\title{
Gestión de la creatividad en la Gerencia de las Cooperativas de Ahorro y Crédito del Ecuador
}

\author{
Chicaiza Taipe, Edgar Germanico* \\ https://orcid.org/0000-0001-7997-3363 \\ Razo Ascazubi, Clara de las Mercedes* \\ https://orcid.org/0000-0002-6418-9549 \\ Paredes Vásquez, Jessica Nataly* \\ https://orcid.org/0000-0002-3435-8057 \\ *Universidad Técnica de Cotopaxi, Latacunga, Ecuador \\ E-mail:edgar.chicaiza1119@utc.edu.ec
}

Recibido: 2 de junio de 2020 Aprobado: 9 de junio de 2020

\section{Resumen}

La gestión de la creatividad en las instituciones financieras se considera uno de los principales pilares de la competitividad en el siglo XXI. El artículo se presenta orientado hacia las Cooperativas de ahorro y crédito del Ecuador, cómo éste afecta el manejo de la gerencia y el desarrollo en el mercado. El objetivo del estudio fue evidenciar los posibles cambios, modificaciones que deben realizar en las entidades financieras, para adaptarse a los nuevos escenarios, evitando el riesgo de estancarse o desaparecer. La investigación se trabajó bajo un enfoque cualitativo, basado en un estudio documental, se integra una perspectiva multidisciplinaria de la toma de decisiones, es decir dentro de las Cooperativas de ahorro y crédito del Ecuador existe una deficiencia por parte de los gerentes, no aplican ideas y habilidades estratégicas para resolver y solucionar problemas, que permitan construir y aprovechar oportunidades para el progreso de la institución, para generar nuevas alternativas e ideas innovadoras dentro de la gerencia. Una adecuada gestión involucra la eficiencia del capital humano garantizando el éxito de la entidad, con resultados de rentabilidad y sostenibilidad en el tiempo.

Palabras clave: Gerencia, Creatividad, Cooperativa de ahorro y crédito. 


\section{Creativity management in the management of Ecuador's Credit Union}

\section{Resumen}

Managing creativity in financial institutions is considered one of the main pillars of competitiveness in the 21st century. The article is presented oriented towards the savings and credit cooperatives of Ecuador, how this affects the management of management and development in the market. The objective of the study was to show the possible changes, modifications that must be made in financial institutions, to adapt to new scenarios, avoiding the risk of stagnation or disappearing. The research was carried out under a qualitative approach, based on a documentary study, a multidisciplinary perspective of decision-making is integrated, that is, within the savings and credit cooperatives of Ecuador there is a deficiency on the part of the managers, they do not apply ideas and strategic skills to solve and solve problems, which allow building and taking advantage of opportunities for the progress of the institution, to generate new alternatives and innovative ideas within the management. Proper management involves the efficiency of human capital, guaranteeing the success of the entity, with results of profitability and sustainability over time.

Keywords: Management, Creativity, Credit union.

\section{Introducción}

La creatividad si bien es cierto no es un término reciente se lo ha visto con un gran desarrollo y reconocimiento en los últimos años, considerando el impulso que se ha dado en el sector empresarial con la finalidad de enfocarse en términos de innovación, expansión, crecimiento y mercado, sin embargo, su concepto se fundamentó desde el mismo inicio de la humanidad y su desarrollo. Si se toma en cuenta como una base el paso que se dio desde la era industrial hacia la era de la información, y cómo ésta tuvo una transformación en la visión de la sociedad dándole importancia profunda a la adquisición del conocimiento; lo que implicó un cambio en la visión del mundo y de cómo el hombre tuvo que adaptarse a situaciones, lugares y enfoques tanto de comportamiento como empresariales.

Es aquí que se observa un cambio radical e interviene significativamente la creatividad buscando alternativas de desarrollo en sectores empresariales, informativos, de comunicación y de aprendizaje. Como menciona Soler hay límites determinados para el uso del vocablo en cuanto a creatividad, pero esas mismas fronteras son vagas y como el tema de creatividad es amplio, los fines entonces pueden ser imprecisos, pero no implica que no se pueda reconocer y trabajar dentro de ellos. (Soler, 2015, p. 31)

Como se mencionó la creatividad ha existido desde la creación del hombre y desde entonces ha tenido una gran evolución en diferentes contextos, en la actualidad en las instituciones se considera indispensable para desarrollar un trabajo efectivo, aporta al crecimiento de las personas, y la innovación en empresas que generan cambios que les permitan alcanzar el éxito.

En ese contexto, en el artículo desarrollado se expondrá a la gestión empresarial de las Cooperativas de Ahorro y Crédito y sus definiciones asociadas a la creatividad y cómo se ha reflejado en características y acciones, siendo ésta un elemento de inspiración hacia la búsqueda de soluciones para crear nuevos movimientos, nuevas alternativas y nuevos incentivos. Es así que éste estudio se enfocó en evidenciar cuál es el problema en el manejo de la gerencia, y cómo impide un crecimiento económico del país, siendo su finalidad el mostrar los posibles cambios y modificaciones que deben realizar en las entidades financieras, para adaptarse a los nuevos escenarios, evitando el riesgo de estancarse o desaparecer. 
Gómez parte de abordar el estudio de la gestión gerencial en las organizaciones en el siglo XXI desde un concepto de desafío, debido a las condiciones que han originado retos en el campo de las ciencias sociales y gerenciales. Donde la postmodernidad trae consigo que los gerentes se enfrenten a escenarios variantes que circundan distintos elementos $\mathrm{y}$ fenómenos, los cuales debe afrontar para mantener sus organizaciones. (Gómez, 2015, p.70)

Se puede evidenciar que la gerencia ha sufrido una evolución basado en el desarrollo tecnológico, innovación y creatividad por los cambios presenciados esencialmente en el siglo XXI, principalmente aportes a la administración de las entidades, en el año 2020 existen empresas modernizadas, los trabajos se los realiza mediante la utilización de medios tecnológicos, las reuniones y toma de decisiones, entre otras cosas. Es importante considerar el análisis que el desarrollo de la creatividad ha tenido en gerentes, en instituciones públicas, privadas y financieras, y la innovación de nuevos productos y actividades para cumplir con objetivos.

\section{Definiendo a la creatividad}

En el amplio espectro conceptual de creatividad se explican en diversas formas las características relacionadas al tema, sin embargo, se pueden desglosar principalmente tres enfoques importantes como es el de producción o empresarial, pensamiento y personalidad. Como es el tema central el de producción, su perspectiva se deriva como generar un producto, definiéndolo como la destreza de generar un trabajo que se pueda adaptar a circunstancias específicas.

Morales (2017), nos dice que "La creatividad se define como la habilidad de producir un trabajo que es, a la vez, novedoso, original o inesperado, y apropiado, útil o adaptativo" (p. 56).

En el desarrollo del artículo se presenta temas fundamentales relacionados con la premisa básica de la habilidad de plantear acciones creativas, sustentada en conceptos y fundamentos teóricos aplicados al ámbito empresarial basado en los elementos teóricos de la temática.

\subsection{Cooperativas de ahorro y crédito del Ecuador}

Las cooperativas de ahorro y crédito ecuatorianas son un elemento vital del sistema financiero del país; han permanecido en el mercado financiero por más de treinta años y, han superado crisis de toda índole. (Carrillo et., 2019, p. 2)

Las entidades financieras se enfocan a ofertar productos financieros, estas gestiones son esenciales para el funcionamiento y crecimiento, si no existiera cada una de las actividades dentro de la entidad no podría alcanzar el éxito dentro del mercado.

\subsection{Creatividad en la toma de decisiones}

La creatividad en la toma de decisiones son componentes que se debe considerar como genérica o transversal, ayudando a la formación de profesionales en los distintos campos del conocimiento, porque al momento de tomar una decisión desarrolla cosas novedosas. (Hernández et., 2015). La creatividad en la toma de decisiones se ha considerado indispensable en la actualidad, ya que permite evaluar alternativas que permitan dar soluciones a situaciones, comprender y enfocarse más a fondo en cada uno de los problemas.

\section{3.- Liderazgo creativo}

El lídercreativo, es aquel que ha sabido aprovechar los conocimientos, valores, habilidades, destreza, entre otros. A lo largo de toda su evolución de vida, donde le ha permitido desarrollar y consolidar una estructura mental con libertad de pensamiento, que nunca se deja llevar por la dependencia de un paradigma sobre sus acciones. Por lo que le da el privilegio de fácil adaptabilidad a sus entornos cambiantes. (Vivolo, 2015, p. 4)

En la organización se debe tener líderes creativos, donde pondrán en práctica habilidades y conocimientos desarrollados en base a la tecnología, el siglo XXI se caracteriza por crear ideas innovadoras, con el fin de mejorar la productividad 
de los trabajadores y ventajas competitivas done se refleja de una forma positiva, para poder cumplir las metas trazadas y alcanzar al éxito.

\section{4.- Gerencia}

La gerencia en una entidad es importante, dentro de ella se desarrollan destrezas y habilidades que cumplen múltiples funciones y coordinan todos los recursos mediante proceso de planeamiento, organización, dirección y control a fin de lograr metas y objetivos establecidos. "Es aquel proceso que se lleva a cabo a través de un individuo que tenga un perfil competencial, en base a sus destrezas, técnicas administrativas y a la comprensión de las relaciones humanas" (Pérez \& Ysaura, 2015, p. 58).

\section{5.- Gestión}

La gestión en una institución es fundamental, asigna responsabilidades sobre un proceso, es necesario considerar que los recursos son importantes al momento de coordinar las actividades con el fin de conseguir y aumentar los resultados óptimos de una empresa.

Es el conjunto de actuaciones que posibilitan la realización de un plan o parte del mismo en una organización y entidad. Los gestores se limitan a la ejecución y su capacidad de decisión está limitada y subordinada a los directivos, aunque a veces es difícil separar estas funciones. (Pérez \& Ysaura, 2015, p. 58)

\subsection{Componentes de la creatividad}

Cada uno de los componentes de la creatividad son importantes para que las Cooperativas de ahorro y crédito gestionen ideas innovadoras acorde a los problemas que pueden ocurrir, la participación de los colaboradores es indispensable debido que las habilidades, talentos y estrategias pueden ser generadas por ellos, para realizar un trabajo exitoso.

\section{- Competencia.}

Procesos relativos a la creatividad: van a depender del entrenamiento, las características de la personalidad y la experiencia en generar ideas.

\section{- Habilidades creativas}

Habilidades relativas al dominio: van a depender de las habilidades cognitivas innatas, perceptivas y motoras además de la educación formal y no formal. Y van a incluir los conocimientos que se tengan sobre el dominio, habilidades técnicas y el talento especial para el dominio específico

\section{- Motivación de la tarea}

Depende del nivel inicial hacia la tarea, si hay presencia de externa y la habilidad cognitiva para evitar el constreñimiento externo. Va a incluir la actitud hacia la tarea y la propia percepción sobre la motivación para comenzar la tarea. (Hernández, 2017, p. 88)

- El conocimiento es clave para mantenerse competitivo y la creatividad añade valor al conocimiento. Las empresas cada vez son valoradas más por su conocimiento que por los activos físicos que poseen. El conocimiento no consiste en información "cruda", sino que se trata de la conexión no discontinua de información que da lugar a ideas y que se convierten en valor. Este proceso se da gracias a la utilización creativa del conocimiento, que convierte información dispersa en valor. (Jiz, 2015, p. 30)

\section{7.- Gestión eficiente}

Una gestión eficiente de los procesos, es aquella que, logra optimizar los recursos, aprovecha las cooperaciones mutuas de la gestión beneficiando a la empresa a mejorar su competitividad y toma de decisiones, todo esto cuidando y preservando el medio en el que actúan, priorizando siempre la salud ocupacional de sus trabajadores. (Bocanegra, 2017)

Una gestión eficiente es importante dentro de una organización, ya sea pública o privada, diariamente tienen que desarrollar la creatividad para mejorar la competitividad y generar eficacia en cada uno de los empleados.

\section{8.- Marketing Digital}

En las Cooperativas de ahorro y crédito, el marketing digital ha tomado gran importancia, 
existen cambios radicales en avances tecnológicos, los gerentes deben realizar estrategias digitales, poniendo en práctica los conocimientos, en una forma más planificada y estructurada con la finalidad de atraer más clientes y mantenerlos satisfechos.

Las instituciones financieras han mejorado sus servicios creando aplicaciones que permiten optimizar el tiempo de los usuarios y realizar diferentes transacciones desde cualquier lugar, a través de la banca móvil, billetera electrónica, entre otros. Existen diferentes herramientas de marketing digital que facilita la difusión de productos financieros.

\section{9.- La creatividad e innovación}

En el siglo XXI la transformación, se ha caracterizado por la incertidumbre y cada vez más diverso e incontrolable, la creatividad es la capacidad que tiene el ser humano de pensar cosas nuevas y ponerlo en ejecución, mientras que la innovación es la capacidad de implementar dichas ideas de un modo diferente. Sin embargo, dentro de las gerencias esto se transforma en un reto que diariamente lo deben sacar adelante, fusionando las dos una organización encaminada por el gerente podrá salir adelante resolviendo todos los obstáculos y las limitaciones que existen. (Hernández et., 2015)

La creatividad e innovación son esenciales dentro de las cooperativas de ahorro y crédito del Ecuador, generando ideas, alternativas y soluciones que les permitan ser competitivos en el mercado, mediante el componente mencionado anteriormente las instituciones pueden alcanzar el éxito en diferentes contextos.

\section{Metodología}

Se debe citar que la metodología tiene como objetivo la captación de información real, que permite a los investigadores realizar un análisis de la conducta, situación o evento para conocer cuál es la causa o motivo para que las personas de ese entorno o lugar mantengan dicha postura, también estos datos recopilados pueden brindar un conocimiento válido siempre y cuando esta posea suficiente fuerza aclarativa. Es por esta razón que se plantea una investigación con enfoque cualitativo que permita utilizar la recolección de información, a través de conceptos, definiciones y aportes sobre las categorías establecidas en el estudio sobre Gestión de la creatividad en la gerencia, de las cooperativas de ahorro y crédito del Ecuador, para establecer conclusiones que contribuyan en la profundización del tema y definición en los procesos de interpretación desde diferentes puntos de vista.

La investigación presentada en este artículo se basa en una recopilación bibliográfica, utilizando una revisión y análisis exhaustiva de textos científicos relacionados con el tema; realizando una revisión documental en la que los investigadores utilizaron varias fuentes de consulta, que constituyen herramientas necesarias para una indagación, algunos de ellos son: libros y revistas de impacto científico, que garanticen el rigor de los resultados. Este tipo de investigación facilita la recopilación de datos en diferentes documentos y fuentes bibliográficas, permite obtener información más amplia, el conocimiento que se construye a partir de la lectura de todos estos documentos.

A través de la investigación cualitativa el investigador busca un concepto que pueda englobar parte de la realidad, no se busca la exactitud sino más bien el entendimiento profundo sobre el tema que se está investigando, en términos generales se parte de lo que dice y hace un individuo, tanto en el ámbito social y cultural, permite comprender la experiencia vivida por las personas. Como mencionan los autores a estos hallazgos no se llegan por medio de procedimiento estadísticos $\mathrm{u}$ otros medios de cuantificación. Puede tratarse de distintas investigaciones con objetivos múltiples como la vida de la gente, experiencias vividas, comportamientos, emociones y sentimientos, así como al funcionamiento organizacional, movimientos sociales, fenómenos culturales y la interacción entre las naciones. (Staruss \& Corbin, 2014, p. 12)

A continuación, se muestra en la Tabla 1 las áreas de investigación trabajadas que se pondrá en evidencia en el estudio. 
Tabla 1

Resumen de las áreas temáticas, categorías, subcategorías y dimensiones

\begin{tabular}{|c|c|c|}
\hline Área Temática & Categorías & Subcategorías \\
\hline \multirow[t]{2}{*}{$\begin{array}{l}\text { Gerencia creativa en } \\
\text { los niveles de la } \\
\text { organización }\end{array}$} & $\begin{array}{l}\text { Definición de gerencia } \\
\text { creativa } \\
\text { Entorno y } \\
\text { creatividad } \\
\text { La creatividad } \\
\text { en la esfera empresarial }\end{array}$ & \\
\hline & $\begin{array}{l}\text { Trabajo en } \\
\text { equipo y creatividad }\end{array}$ & $\begin{array}{l}\text { Ventajas de los equipos de } \\
\text { trabajo }\end{array}$ \\
\hline \multirow{3}{*}{$\begin{array}{l}\text { Creatividad } \\
\text { Empresarial: proceso } \\
\text { creativo de generación } \\
\text { de ideas }\end{array}$} & Proceso creativo & $\begin{array}{l}\text { Generación y selección de } \\
\text { ideas. }\end{array}$ \\
\hline & La actitud creativa & $\begin{array}{l}\text { Considerar las ideas de } \\
\text { todo el personal. }\end{array}$ \\
\hline & $\begin{array}{l}\text { Creación de un entorno } \\
\text { creativo en la empresa }\end{array}$ & $\begin{array}{l}\text { Formar a los trabajadores } \\
\text { en técnicas creativas. }\end{array}$ \\
\hline La gerencia estratégica. & $\begin{array}{l}\text { Niveles de la } \\
\text { organización }\end{array}$ & $\begin{array}{l}\text { Nivel Estratégico } \\
\text { Nivel táctico } \\
\text { Nivel operativo }\end{array}$ \\
\hline \multirow[t]{2}{*}{$\begin{array}{l}\text { Gerencia integral } \\
\text { creativa }\end{array}$} & Capacidad de gestión & $\begin{array}{l}\text { Liderazgo creativo } \\
\text { Resolución creativa de } \\
\text { retos. } \\
\text { Toma de decisiones. }\end{array}$ \\
\hline & $\begin{array}{l}\text { Capacidad de } \\
\text { organización }\end{array}$ & $\begin{array}{l}\text { Participación creativa. } \\
\text { Integración creativa. } \\
\text { Comunicación creativa. }\end{array}$ \\
\hline
\end{tabular}

Fuente: Adaptación de: Gerencia creativa en el Instituto Pedagógico de Caracas. Sol Ángel Martínez. 2013.

\section{Resultados}

\subsection{Gerencia estratégica}

Al hablar de Gerencia estratégica es importante definir que es un proceso que permite al gerente ser proactivo, facilita la evaluación de las acciones que orientan al logro de los objetivos organizacionales, cabe manifestar que el gerente no solo debe actuar a nivel estratégico, sino también debe estar involucrado en los niveles táctico y operativo.

La Gerencia Estratégica, es una herramienta para administrar y ordenar los cambios, donde se definen los objetivos de la organización y se establecen estrategias; así mismo, se reconoce la participación basada en el liderazgo y la toma de decisiones que correspondan a las demandas del ambiente inmediato y futuro. (Gonzáles et ál., 2019, p. 244)

La dirección táctica en una cooperativa está representado por los jefes de cada departamento, sean estos financieros o de cobranzas, responsables de realizar acciones para hacer realidad la estrategia de la empresa; mientras que en la dirección operativa intervienen las personas que hacen labor operativa, como por ejemplo las cajeras y asesores de crédito, que son los responsables de ejecutar las acciones desarrolladas en el nivel táctico; sin embargo, el gerente como el responsable de dirigir la cooperativa debe ser partícipe en cada una de las actividades desarrolladas en los niveles mencionados anteriormente, consecuentemente se toma decisiones 
conjuntas que permitan obtener rentabilidad y un mejor desempeño de sus colaboradores.

\subsection{Creatividad empresarial}

La creatividad empresarial no depende solamente de los gerentes de las Cooperativas de Ahorro y Crédito, que son los encargados de generar y asegurar la fidelidad de los clientes, la creatividad debe verse reflejado en los empleados y trabajadores de la institución financiera, que son consideradas como técnicas atractivas e ingeniosas que tienen como objetivo incidir en los aspectos del pensamiento, para mejorar el rendimiento laboral.

La creatividad para una empresa es la innovación y explotación de nuevas posibilidades para atraer clientes, se debe hacer todo lo posible para promover la creatividad al interior de los negocios, pues ésta es la principal estrategia que permitirá asegurar la fidelidad de los clientes. (Herrera \& Duarte, 2016, p. 7)

Es necesario identificar, generar, seleccionar y aplicar las mejores alternativas con aspectos fundamentales de una actitud positiva e innovadora, para lograr este cambio en una cooperativa, es de vital importancia que las ideas y opiniones de los colaboradores sean creativas.

\subsection{Gerencia creativa}

El gerente debe basarse en un liderazgo creativo que permita afrontar la complejidad, en el caso de las cooperativas la cobranza es compleja, debido al incremento del desempleo generado por la crisis económica que atraviesa el país, por ende es necesario contar con un líder que motive al talento humano a asumir riesgos y enfrentar retos, que haga participar a sus trabajadores, para que se sientan bien en su lugar de trabajo, a pesar de las dificultades que deben afrontar; del mismo modo la institución debe estar preparada a vivir con incertidumbre.

\subsection{Generación de ideas}

La generación de ideas no debe surgir solo de la gerencia, sino también de los niveles táctico y operativo, por la relación que existe entre los mismos, las ideas creativas aportan a la solución de problemas y conflictos de manera inmediata, contribuye a que los procesos de la cooperativa sean más competitivos para ampliar la oferta de un servicio de excelencia a los usuarios. Las ideas creativas pueden venir del personal de limpieza, asistentes, cajeros, jefes departamentales, asesores de crédito, entre otros, porque una habilidad que cualquier persona la puede desarrollar en un momento determinado.

\subsection{Gerencia integral creativa}

La gerencia integral creativa es la capacidad que poseen los jefes o gerentes de una cooperativa $o$ institución financiera para planear y dirigir con el uso de los diferentes tipos o modos de pensamiento de sus colaboradores, con una capacidad de gestión y orientación a mantener un determinado direccionamiento o propósito a conseguir a través de sus propios aportes hacia la organización.

\subsection{Toma de decisiones}

Para realizar una correcta y oportuna toma de decisiones creativas en una cooperativa, es inevitable que se puedan descubrir las limitaciones y malas prácticas que se están utilizando, para que a través de este proceso se adapten las mejores ideas que pueda aportar el personal siempre buscando la innovación y el adaptarse a los cambios tecnológicos que con el tiempo siempre se presentan, pero con una actitud creativa cualquier tipo de problemática se vuelve una oportunidad de desarrollo para la empresa.

\subsection{Liderazgo creativo}

Un líder que posea una actitud creativa permite en las instituciones financieras, como las cooperativas, puedan prever y desarrollar sus actividades en un campo competitivo, mejorando su nivel de liquidez para satisfacer las necesidades tanto de sus colaboradores como de sus clientes, al mantener comportamientos y actitudes, como el entusiasmo y principalmente brindar un buen servicio, que son claves para optimizar la imagen corporativa. 


\section{Discusión}

Los principios de este estudio claramente demuestran que la creatividad ha tenido una evolución dentro de las instituciones financieras, permite a los gerentes generar nuevas ideas y proponer nuevas estrategias como la banca móvil, billetera electrónica, asesoría de Pymes y redes sociales que facilite la toma de decisiones, consecuentemente la institución podrá adaptarse a las nuevas tendencias de consumo de los clientes dentro de un mercado competitivo.

Una explicación para este cambio se da por la evolución mercantil en un mundo globalizado y toda gira en torno a los cambios que se producen, todas las gestiones realizadas por los gerentes dentro de las instituciones financieras requieren de nuevas ideas y creatividad que permitan enfrentar el reto de generar confianza con los clientes ofertando un servicio y atención al cliente de calidad. El gerente debe mejorar la comunicación en los niveles tácticos y operativos, modernizando las áreas de trabajo de cada uno de los funcionarios de las cooperativas de ahorro y crédito, con la finalidad de fomentar el trabajo en equipo.

\subsection{Internet vs televisión}

En el siglo XXI la mayoría de personas optan por utilizar el internet, con un promedio diario de 170 minutos para revisar las publicidades que ofertan las empresas e instituciones financieras, mientras que el consumo de la televisión es de 167 minutos diarios, resultados que reflejan una preferencia por el marketing digital, herramienta que permite ganar nuevos clientes.

El consumo total de medios (televisión, radio, internet, entre otros) ha alcanzado hasta el momento un promedio de ocho horas diarias por persona. Además de logar un record en la cantidad de tiempo dedicado al consumo de medios, 2019 será recordado como el año en el que internet destronó, por primera vez en la historia, a la televisión como plataforma estrella de los medios de comunicación. (Media, 2019)

\subsection{Coworking}

El gerente debe mantener una comunicación efectiva con los niveles tácticos y operativos que permita tomar decisiones en beneficio de la cooperativa, utilizan estrategias como el coworking que es una modalidad de trabajo que facilita a los funcionarios de una institución financiera compartir un mismo espacio para trabajar en equipo para alcanzar de manera efectiva y eficiente los objetivos planteados.

El coworking es la implementación de un espacio físico en donde por medio de un modelo cooperativo se puede obtener por un bajo costo el espacio o los insumos necesarios para poder realizar una actividad económica en un ambiente colaborativo y en conjunto con una amplia gama de profesionales y contactos. (Peñafiel, 2018, p. 754)

\section{Conclusiones}

La gestión de ideas creativas en la gerencia, cumple un rol importante para alcanzar los objetivos estratégicos en las entidades financieras. Las ideas creativas permiten afrontar adversidades, adaptar a los cambios que facilite la solución inmediata de los problemas que se suscitan al interior de una entidad financiera, pueden surgir del personal de limpieza, asistentes, cajeros, jefes departamentales, asesores de crédito, entre otros, porque es una habilidad o talento que cualquier persona la puede desarrollar en un momento determinado.

La gerencia creativa es la capacidad que poseen los gerentes de una institución financiera donde generan, fomentan y potencializan la creatividad en el trabajo, de modo que los lleve a mantener ventajas competitivas y mantener una relación de largo plazo con los clientes. Es necesario determinar que estos procesos requieren de elementos de trabajo en equipo y personal para que las propuestas sean de valor. El gerente creativo no debe temer a equivocarse, porque es una oportunidad para aprender y afrontar nuevos retos, se debe buscar nuevas estrategias que permiten la continuidad y el crecimiento financiero. 
El marketing digital y el coworking son estrategias utilizadas por los gerentes de las cooperativas de ahorro y crédito, con la finalidad de mejorar el ambiente laboral en el que todos los miembros de la institución aporten con ideas creativas y fomenten el trabajo en equipo; las plataformas virtuales son medios utilizados para ofertar o dar a conocer los productos y servicios financieros, los clientes se mantienen informados sobre las novedades o actualizaciones que realizan frecuentemente las instituciones financieras.

\section{Referencias bibliográficas}

Bocanegra, Bocanegra, C. (2018). La innovación en la gestión eficiente de la Municipalidad de San Borja en el año2017http://repositorio. ulasamericas.edu.pe/bitstream/handle/upa/341/ TESIS $\% 20$ FINAL $\% 20$ BOCANEGRA $\% 20$ -13-06-2018.pdf?sequence $=1 \&$ isAllowed $=\mathrm{y}$

Cadena, P.; Rendón, R.; Aguilar, J.; Salinas, E.; de la Cruz, F y Sangerman, D. (2017). Métodos cuantitativos, métodos cualitativos o su combinación en la investigación: un acercamiento en las ciencias sociales. Revista Mexicana de Ciencias Agrícolas. 8(7), 1603-1617. https://www.redalyc.org/ pdf/2631/263153520009.pdf

Carrillo, A.; Galarza, S.; Guayasamín, C. (2019). Características Culturales entre una Cooperativa de Ahorro y Crédito Ecuatoriana y el sector cooperativo Nacional 10(2), 3-12. https://revistas.ute.edu.ec/index.php/economiay-negocios/article/view/615/477

Dugarte, E. (2016).. Tipos y paradigmas M_D. Consultado el 9 de septiembre de 2020. http:// invdocumb2016.blogspot.com/2017/01/

Gómez, E. (2015) Gestión Gerencial de la creatividad en organizaciones innovadoras. Revista

ASA, 68 - 84. http://revencyt.ula.ve/storage/repo/ ArchivoDocumento/asa/n5/art06.pdf

Gonzáles, J.; Salazar, F.; Ortíz, R. y Verdugo, D.
(2019). Gerencia Estratégica: Herramienta para la toma de decisiones en las organizaciones. TELOS, 21(1), 242-267. http://ojs.urbe.edu/ index.php/telos/article/view/3002

Hernández, A. (2017). Un Recurso de Innovación para Docentes: Programa "Despierta Creatividad"https://www.tdx.cat/bitstream/ handle/ $10803 / 482082$ /TAFHO.pdf? sequence $=1$

Hernández, A.; Alvarado, J. \& Luna, S. (2015). Creatividad e innovación: competencias genéricas o transversales en la formación profesional. (44), 135-151.https://www.redalyc. org/pdf/1942/194238608010.pdf

Herrera, M.; Duarte, Y. (2016). Creatividad empresarial: Automatización del proceso de ventas, Universidad Nacional Autónoma de Nicaragua, Managua, https://repositorio.unan. edu.ni/3609/1/17038.pdf

Jiz, L. (2015). "La Creatividad En Los Mandos Medios De Distribuidora Huehueteca, S. A."

http://recursosbiblio.url.edu.gt/ tes is j cem/2015/05/43/Jiz-Linda. pdfMarketingdigital. (2018). El Marketing Digital. https://www.mdmarketingdigital.com/ que-es-el-marketing-digital.php

Media, Z. (2019). Consumo de los medios de comunicación en Europa. [Mensaje en un blog]. Statista. https://es.statista.com/grafico/14411/ el-consumo-de-internet-sobrepasa-al-de-la-tv/

Morales, C. (2017). La creatividad una revisión científica. Arquitectura y Urbanismo 39(2), 53-62. https://www.redalyc.org/ pdf/3768/376852683005.pdf

Peñafiel, A. (2018). Coworking, una alternativa de mejora la subempleo en Babahoyo. Revista digital de Ciencia, Tecnología e Innovación, 5 (Número especial), 751-761.file://C:/Users/ HP/Desktop/1524-4799-1-PB\%20coworki.pdf

Pérez, Ysaura. (2015). La Gerencia De Finanzas 
en el manejo de la administración pública de una institución educativa gubernamental del estado Aragua (Inces)http://mriuc.bc.uc.edu. ve/bitstream/handle/123456789/2099/yperez. pdf?sequence $=1$

Portilla, M.; Rojas, A.; Hernández, I. (2014). Investigación cualitativa: una reflexión desde la educación como hecho social. Artículo - línea de investigación: teorías y procesos curriculares. 3(2), 86-100. file:///C:/Users/ HP/Downloads/2 192-Texto\%20de1\%20 art\%C3\%ADculo-7372-2-10-20150826.pdf

Rovira, A. (2015). Equipos y talento. http://www. equiposytalento.com/noticias/2015/02/13/eltrabajo-en-equipo-es-lafuente-del-exito.

Soler, M. (2015). La Creatividad como Perspectiva en la Formación Académica Universitaria. [Tesis Doctoral Estudio de los ingresantes a la Facultad de Ciencias Agrarias, Universidad Nacional de Jujuy]. https://digitum.um.es/digitum/ bitstream/10201/48361/1/Mar\%C3\%ADa\%20 Sof $\%$ C3\%ADa $\% 20$ Soler $\% 20$ Pasquini $\% 20$ Tesis\%20Doctoral.pdf

Vivolo, C. (2015). Liderazgo creativo en los Directores Educativos del siglo XXI. Omnia, 21(1), 129-141. https://www.redalyc.org/ pdf/737/73742121010.pdf

Staruss, A. \& Corbin, J. (2014). Bases de la investigación cualitativa ( $2^{\mathrm{a}}$ ed). Editorial Universidad de Antoquia. https://books.google.com. ec/:\%22Juliet + Corbin $\% 22 \& h l=e s \& s a=X \& v e d$ $=2$ ahUKEwjv

AEwAHoECAYQAg\#v $=$ onepage $\& q \& \mathrm{f}=$ false 